\title{
Disease- and age-related changes in histone acetylation at gene promoters in psychiatric disorders
}

\author{
B Tang ${ }^{1}$, B Dean ${ }^{2,3}$ and EA Thomas ${ }^{1}$
}

Increasing evidence suggests that epigenetic factors have critical roles in gene regulation in neuropsychiatric disorders and in aging, both of which are typically associated with a wide range of gene expression abnormalities. Here, we have used chromatin immunoprecipitation-qPCR to measure levels of acetylated histone H3 at lysines 9/14 (ac-H3K9K14), two epigenetic marks associated with transcriptionally active chromatin, at the promoter regions of eight schizophrenia-related genes in $n=82$ postmortem prefrontal cortical samples from normal subjects and those with schizophrenia and bipolar disorder. We find that promoter-associated ac-H3K9K14 levels are correlated with gene expression levels, as measured by real-time qPCR for several genes, including, glutamic acid decarboxylase 1 (GAD1), 5-hydroxytryptamine receptor 2C (HTR2C), translocase of outer mitochondrial membrane 70 homolog A (TOMM70A) and protein phosphatase 1E (PPM1E). AC-H3K9K14 levels of several of the genes tested were significantly negatively associated with age in normal subjects and those with bipolar disorder, but not in subjects with schizophrenia, whereby low levels of histone acetylation were observed in early age and throughout aging. Consistent with this observation, significant hypoacetylation of H3K9K14 was detected in young subjects with schizophrenia when compared with age-matched controls. Our results demonstrate that gene expression changes associated with psychiatric disease and aging result from epigenetic mechanisms involving histone acetylation. We further find that treatment with a histone deacetylase (HDAC) inhibitor alters the expression of several candidate genes for schizophrenia in mouse brain. These findings may have therapeutic implications for the clinical use of HDAC inhibitors in psychiatric disorders.

Translational Psychiatry (2011) 1, e64; doi:10.1038/tp.2011.61; published online 20 December 2011

\section{Introduction}

Epigenetic mechanisms of gene regulation involve both DNA methylation and posttranslational modifications of histone proteins. ${ }^{1}$ Although it is known that DNA methylation of cytosine residues at $\mathrm{CpG}$ dinucleotide sites results in gene silencing, the effects of posttranslational modifications on histone proteins are more complex. ${ }^{2}$ Histone tails are subjected to many kinds of chemical modifications, such as methylation, acetylation, phosphorylation, ubiquitination and ribosylation, ${ }^{3}$ which can lead to diverse effects on chromatin structure and gene activity. For example, acetylation of lysine residues usually correlates with chromatin accessibility and transcriptional activation, whereby lysine methylation has either activating or repressive effects on gene regulation. ${ }^{3}$

During the last several years, there has been an increased interest in the epigenetic origins of psychiatric diseases. ${ }^{4-7}$ Of the diverse epigenetic machinery associated with gene regulation, DNA methylation has been the most widely studied in the context of psychiatric disorders. Altered methylation status of CpG sites has been found within the regulatory regions of several candidate genes in subjects with schizophrenia, including $H T R 1 A,{ }^{8} H T R 2 A,{ }^{9}$ glutamic acid decarboxylase 1 (GAD1), ${ }^{10,11}$ REELIN, ${ }^{12,13}$ COMT, ${ }^{14} D R D 2^{15}$ and SOX10. ${ }^{16}$ More recently, epigenome-wide profiling has revealed large scale changes in DNA-methylation associated with major psychosis, some of which involve genes associated with neuronal development as well as genes involved with glutamatergic and GABAergic neurotransmission. ${ }^{17}$

To date, much less is known about alterations in histone modifications in schizophrenia. Previous studies have quantified global levels of histone phosphorylation, acetylation and methylation, at different lysine $(K)$, serine $(S)$ and arginine $(R)$ residues of histones $\mathrm{H} 3$ and $\mathrm{H} 4$. Overall, no significant differences in the levels of these histone marks were found in the prefrontal cortex of individuals with schizophrenia compared with normal control subjects; ${ }^{18}$ however, higher methylation levels of histone $\mathrm{H} 3$ at R17 were detected within a subset of affected patients. ${ }^{18}$ More recently, decreases in trimethylated $\mathrm{H} 3$ at $\mathrm{K} 4$ were found specifically at the GAD1 locus in the prefrontal cortex of patients with schizophrenia compared with control subjects, in correlation with reduced GAD1 mRNA levels. ${ }^{11}$ These data suggest that changes in histone modifications at specific genomic loci, rather than on a global scale, may be occurring in schizophrenia, and identification of such loci may unveil the role of epigenetic regulation of gene expression in schizophrenia.

Given the widespread changes in gene expression that have been associated with psychiatric disorders, ${ }^{19,20}$ we investigated the contribution of histone acetylation at specific gene promoters to gene expression regulation in schizophrenia and bipolar disorder. Previous studies have shown that acetylation

${ }^{1}$ Department of Molecular Biology, The Scripps Research Institute, La Jolla, CA, USA; ${ }^{2}$ The Rebecca L. Cooper Research Laboratories, The Mental Health Research Institute, Parkville, VIC, Australia and ${ }^{3}$ The Department of Psychiatry, The University of Melbourne, Melbourne, VIC, Australia

Correspondence: Dr EA Thomas, Department of Molecular Biology, The Scripps Research Institute, SP-2030, 3030 Science Park Dr, La Jolla, CA, 92037, USA.

E-mail: bthomas@scripps.edu

Keywords: bipolar disorder; chromatin; epigenetic; HDAC inhibitor; schizophrenia

Received 14 July 2011; revised 4 November 2011; accepted 5 November 2011 
levels of histone $\mathrm{H} 3$ at K9 (ac-H3K9) and at K14 (ac-H3K14) are highest at the predicted transcriptional start sites of active genes and are positively correlated with one another, as well as with transcriptional activity across a range of yeast genes. ${ }^{21}$ Therefore, we measured histone acetylation at $\mathrm{K} 9$ and $\mathrm{K} 14$ at the proximal promoter regions of eight selected genes representing diverse functionalities that have been implicated in the pathophysiology of schizophrenia.

We find that histone acetylation at K9 and K14 is associated with gene expression levels for eight schizophrenia-related genes, and that histone acetylation patterns at specific loci show distinct disease and age-related effects in normal subjects and those with schizophrenia and/or bipolar disorder. Importantly, understanding the role of histone acetylation in schizophrenia and bipolar disorder may have relevant therapeutic implications, whereby the use of histone deacetylase (HDAC) inhibitors may be clinically beneficial by means of restoring abnormal histone acetylation patterns and accompanying gene expression deficits in schizophrenia and with aging in normal subjects.

\section{Materials and methods}

Samples. This study utilizes postmortem human brain samples ( $n=82$ in total) from two different brain banks: The Harvard Brain Tissue Resource Center (HBT) and the Victorian Brain Bank Network (VBBN) at the Mental Health Research Institute. For the VBBN samples, approval was obtained from both the Ethics Committee of the Victorian Institute of Forensic Medicine and the Mental Health Research and Ethics Committee of Melbourne Health. Cases were split into two groups. The first group consists of brains from the HBT collection: the prefrontal cortex (Brodmann area 10) from $n=50$ subjects ( $n=18$ normal subjects, $n=16$ subjects with schizophrenia and $n=16$ subjects with bipolar disorder). The second group consisted of young subjects from the VBBN collection: the prefrontal cortex (Brodmann area 46) from $n=16$ subjects $(n=8$ control, $n=8$ subjects with schizophrenia; $18-36$ years of age) and old subjects from the HBT collection: the prefrontal cortex (Brodmann area 10) from $n=16$ subjects $(n=8$ control and $n=8$ subjects with schizophrenia; 55-92 years of age). Demographic data for individual subjects are shown in Supplementary Table 1. Ascertainment and diagnosis of all subjects were based on the diagnostic and statistical manual of mental disorders (DSM-IV) criteria (American Psychiatric Association 1994). In the case of the VBBN collection, an additional validated instrument, the Diagnostic Instrument for Brain Studies, was used. ${ }^{22}$ None of the subjects had a record of treatment with valproic acid, an HDAC inhibitor. For the VBBN subjects, cadavers had been refrigerated within $5 \mathrm{~h}$ from death to ensure slowing of any autolysis of the CNS tissue; the recorded postmortem intervals (PMIs) include refrigeration times. For these samples, tissue integrity was assessed by $\mathrm{pH}$, which is now recognized as a better measure of tissue preservation than $\mathrm{PMI},{ }^{23}$ and all samples with $\mathrm{pH}<6.1$ were excluded. For all samples, RNA quality was assessed by Bioanalyzer tracings or gel electrophoresis and spectrophotometric measurements, which showed no evidence for degradation products or protein contamination.
Chromatin immunoprecipitation. Chromatin immunoprecipitation (ChIP)-PCR was performed on postmortem human brain samples using an adaptation of a method previously described in detail. ${ }^{24}$ Briefly, $\sim 60-100 \mathrm{mg}$ of the prefrontal cortex from human postmortem brain was fixed with $1 \%$ of formaldehyde for $15 \mathrm{~min}$ at room temperature then homogenized to isolate nuclei. DNA was sonicated in lysis buffer (1\% SDS, 10 mM EDTA, 50 mM Tris-HCl ( $\mathrm{pH} 8.0), 1 \times$ protease inhibitors cocktail (Roche, Germany)) to $\sim 0.2-$ $0.8 \mathrm{~kb}$ in size of DNA fragments. $100 \mu \mathrm{l}$ of precleared nuclear lysate was diluted with dilution buffer $(1 \%$ Triton $\times 100,2 \mathrm{mM}$ EDTA, $20 \mathrm{mM}$ Tris- $\mathrm{HCl}(\mathrm{pH} 8.0), 150 \mathrm{mM} \mathrm{NaCl}$ and $1 \times$ protease inhibitors cocktail), and incubated with $3 \mu \mathrm{g}$ of histone ac-H3K9K14 (Upstate, Billerica, MA, USA), $3 \mu \mathrm{g}$ rabbit control IgG (Cell Signaling Technology, Danvers, MA, USA) or total histone $\mathrm{H} 3$ (Abcam, Cambridge, MA, USA) antibodies overnight at $4{ }^{\circ} \mathrm{C} .60 \mu \mathrm{l}$ of Protein A Agarose beads (Millipore, CA, USA) were added and incubated for $2 \mathrm{~h}$ to capture the immune complexes. The protein-DNA complexes were washed and eluted in elution buffer $(1 \%$ SDS and $0.1 \mathrm{M} \mathrm{NaHCO}_{3}$ ) at $65^{\circ} \mathrm{C}$ for $20 \mathrm{~min}$. The proteins were digested by proteinase $\mathrm{K}$, and the cross-linking reaction was reversed at $65^{\circ} \mathrm{C}$ overnight. DNA was purified with phenol/chloroform and ethanol precipitation, and analyzed by real-time PCR analysis.

Gene expression analysis. Real-time qPCR analysis was performed using the ABI PRISMs 7900HT Sequence Detection System (Applied Biosystems, Foster City, CA, USA) on the recovered DNA from the ChIP experiments using primers directed against the proximal promoter regions of schizophrenia-related genes (Supplementary Table 2), or on cDNA prepared from the same samples using the primers designed in the exonic regions of selected genes (Supplementary Table 2) as described previously. ${ }^{25,26}$ The proximal promoter region ( $\sim 1 \mathrm{~kb}$ upstream from transcription start site) of each gene was obtained from UCSC browser (http://genome.ucsc.edu/cgi-bin/hgGateway). Primers were designed to generate amplicons of 80-150 nucleotides with similar melting temperatures $\left(64^{\circ} \mathrm{C}\right)$ using Invitrogen's Primer Designer and their specificity for binding to the desired sequences was searched against the NCBI database. We analyzed the ChIP-qPCR data using the Percent Input Method (Invitrogen, Carlsbad, CA, USA). Briefly, the amplification efficiency $(A E)$ of the qPCR reaction for each primer pair and sample was determined by the input DNA using the formula $\mathrm{AE}=10^{\wedge}(-1 /$ slope $)$. The threshold cycle $(\mathrm{Ct})$ value of Input, which is $1 \%$ of the immunoprecipitation (IP) reaction was adjusted to $100 \%$ by subtracting 6.644 cycles (log2 of 100 ), and then the percent input was calculated by the formula $100 \times \mathrm{AE}^{\wedge}$ (adjusted input Ct-IP Ct). For gene expression, the amount of cDNA in each sample was calculated using SDS2.1 software (Applied Biosystems, Foster City, CA, USA) by the comparative Ct method and expressed as $2 \exp (\mathrm{Ct})$ using beta-2-microglobulin (B2M) as an internal control.

Differences in the levels of microarray expression values, from our previously published microarray dataset, ${ }^{27}$ were calculated by ANOVA, performed using the National Institutes of Aging Array Tools, ${ }^{28}$ with the FDR controlled at a default setting of 0.1 , according to Benjamini and Hochberg. ${ }^{29}$ 
Statistics. The demographic characteristics for each cohort were compared using Student's $t$-tests to verify matching for age, sex ratio, PMls and tissue $\mathrm{pH}$. The PMI between the two brain banks were significantly different $(46.13 \pm 2.43$ vs $21.3 \pm 1.74 \mathrm{~h} ; P<0.0001$ ) (Supplementary Table 1), which could be due, in part, to different criteria for defining PMI (see above). Importantly, the PMls did not show any significant difference when compared by cohort. The gene expression and ChIP-qPCR data values were analyzed for normal distribution using the Kolmogorov-Smirnov method, which confirmed that the data were normally distributed for all subjects. Given that the data were normally distributed, each data set was interrogated for outliers using the Grubbs' test, which resulted in the removal of ChIP-qPCR values from one of the old control subjects from the HBT collection. For assessment of disease effects of the qPCR and ChIP-qPCR data among the control, schizophrenia and bipolar disorder cohorts, significant differences were determined by one-way ANOVA and Student's unpaired $t$-tests (GraphPad 5.0; San Diego, CA, USA). The effects of demographic and brain collection parameters (age, sex, $\mathrm{PMI}$ and tissue $\mathrm{pH}$ ) on the disease effect for all data were assessed by ANCOVA (XLSTAT software, Addinsoft, New York, NY, USA). From this analysis, age showed a significant contribution to data variation in gene expression and/or ChIP data for all genes tested (Supplementary Table 3). Tissue $\mathrm{pH}$ showed a significant effect on gene expression only for translocase of outer mitochondrial membrane 70 homolog A (TOMM70A) in the schizophrenia comparison and for GAD1 in the bipolar disorder comparison, but no significant effects of $\mathrm{pH}$ on ChIP data were observed for any genes (Supplementary Table 3). Pearson's product moment correlation analysis was further performed for the ac-H3K9K14 levels (as percentage input) and the B2M-normalized expression values against the age of the subjects, and for ac-H3K9K14 levels against the gene expression values.

\section{Results}

Disease effects on gene expression . We selected eight diverse "schizophrenia-related" genes (Table 1) for this study based on the following criteria: (1) genes showing differential expression in schizophrenia and/or bipolar disorder from published microarray studies; ${ }^{30,31}$ (2) genes showing CNS cell type-specific expression patterns based on comparison with previous transcriptome studies performed on isolated astrocytes, neurons and oligodendrocytes; ${ }^{32}$ (3) genes representing different functions/pathways related to schizophrenia based on review of the literature. ${ }^{20,33-35}$ Additionally, these selected genes are representative of different gene co-expression networks, based on our previous studies, which identified over 20 gene coexpression modules in the prefrontal cortex from subjects with schizophrenia and bipolar disorder. ${ }^{36}$ We first tested for expression differences for five neuronally expressed genes, GABAergic neurotransmission: GAD1; mitochondrial function/import: TOMM70A; neurotransmitter receptor signaling: serotonin 5-hydroxytryptamine receptor $2 \mathrm{C}$ (HTR2C) and regulator of G protein signaling 4 (RGS4); signal transduction: protein phosphatase, $\mathrm{Mg}^{2+} / \mathrm{Mn}^{2+}$ dependent, 1E (PPM1E) in the postmortem prefrontal cortex (Brodmann area 10) from a cohort of subjects with schizophrenia and bipolar disorder from the Harvard Tissue Resource Center (group 1; Supplementary Table 1). Realtime qPCR analysis revealed decreased expression of HTR2C, TOMM70A, RGS4 and PPM1E in subjects with schizophrenia and bipolar disorder compared with matched controls, and a decrease expression in GAD1 only in subjects with schizophrenia (Figure 1).

Histone acetylation at gene promoters. To test for correlations between gene expression activity and promoter histone acetylation, we performed ChIP-qPCR assays on cortical samples from these same subjects, using an antibody directed against ac-H3K9K14, followed by realtime qPCR analysis using primers directed against the proximal promoter regions of these genes. Linear regression analysis revealed that gene expression levels were correlated with promoter-associated ac-H3K9K14 levels for GAD1, TOMM70A, HTR2C and PP1ME, but not for RGS4, in all 50 subjects (psychiatric cases and controls) (Figure 2). Ac-H3K9K14 levels were also compared among all psychiatric cases and controls, and no significant differences were detected (data not shown).

Table 1 Summary of genes selected for this study

\begin{tabular}{|c|c|c|c|c|c|c|}
\hline Gene ID & Gene description & Cell type association ${ }^{\mathrm{a}}$ & Function & $s c Z^{b}$ & $S C Z^{c}$ & $B P^{C}$ \\
\hline$G A D 1$ & Glutamic acid decarboxylase 1 & Neuron & GABAergic neurotransmission & $\downarrow$ & $\downarrow$ & I \\
\hline HTR2C & $\begin{array}{l}\text { 5-hydroxytryptamine (serotonin) } \\
\text { receptor } 2 \mathrm{C}\end{array}$ & Neuron & Neurotransmitter receptor signaling & $\downarrow$ & $\uparrow$ & \\
\hline RGS4 & Regulator of G-protein signaling 4 & Neuron & & & - & $\downarrow$ \\
\hline TOMM70A & $\begin{array}{l}\text { Translocase of outer mitochondrial } \\
\text { membrane } 70 \text { homolog A }\end{array}$ & Neuron & Mitochondrial function/import & $\downarrow$ & $\downarrow$ & \\
\hline PPM1E & $\begin{array}{l}\text { Protein phosphatase, } \mathrm{Mg}^{2+} / \mathrm{Mn}^{2+} \\
\text { dependent, } 1 \mathrm{E}\end{array}$ & Neuron & Signal transduction & $\downarrow$ & $\downarrow$ & $\uparrow$ \\
\hline$M B P$ & Myelin basic protein & Oligodendrocyte & Myelination-associated & $\downarrow$ & $\downarrow$ & $\downarrow$ \\
\hline UGT8 & UDP glycosyltransferase 8 & Oligodendrocyte & White matter function & $\downarrow$ & & \\
\hline H1FNT & $\mathrm{H} 1$ histone family, member $\mathrm{N}$ & Ubiquitous & Chromatin-related & $\downarrow$ & NP & NP \\
\hline
\end{tabular}

The arrows designate the direction of the significant gene expression change in schizophrenia (SCZ) or bipolar disorder (BP) from previous microarray studies. '-' designates no significant change in expression; NP, indicates not present in the dataset.

${ }^{a}$ Cell-type specific expression was determined by comparing with transcriptome datasets for astrocytes, neurons and oligodendrocytes, from Cahoy et al. ${ }^{32}$

${ }^{\mathrm{b}}$ From Narayan et al. ${ }^{30}$

${ }^{c}$ From the Stanley Medical Research Database, https://www.stanleygenomics.org/and Kim and Webster. ${ }^{31}$ 
Age effects on gene activity. ANCOVA of the demographic and sample variables with the experimental data values revealed that age significantly contributed to the variation in gene expression and/or ac-H3K9K14 levels among disease cohorts for all genes tested. Therefore, we further highlighted the effects of age on ac-H3K9K14 levels by performing Pearson's linear correlation analyses. Promoter associated ac-H3K9K14 levels were significantly negatively associated with age for GAD1, RGS4, PPM1E,

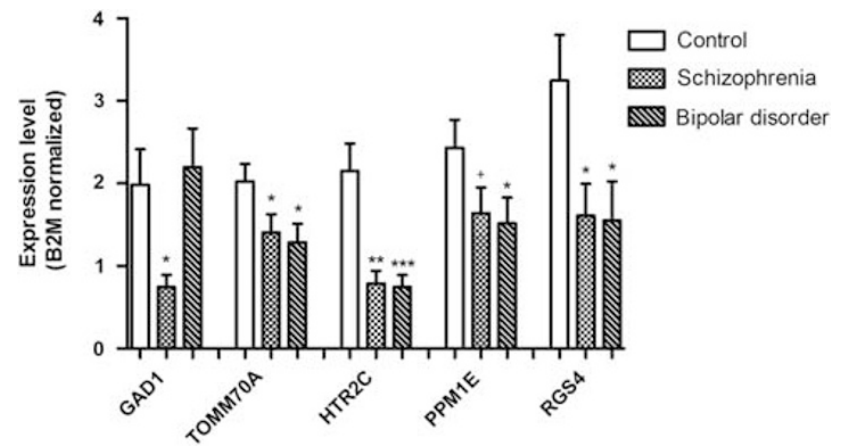

Figure 1 Real-time PCR analysis for the indicated genes in subjects with schizophrenia, bipolar disorder and non-psychiatric controls. Real-time qPCR assays were performed as described in the materials and methods on postmortem Brodmann area (BA) 10 from subjects with schizophrenia, bipolar disorder and matched controls (group 1; $n=50$ total). Data shown are gene expression values normalized by the housekeeping gene, B2M. Asterisks denote significant differences in expression as determined by one-way ANOVA followed by Student's t-test: ${ }^{*} P<0.05 ;{ }^{+} P<0.08 ;{ }^{* \star} P<0.01 ;{ }^{* \star} P<0.001$.
HTR2C and TOMM70A in normal subjects (Table 2) Importantly, there was also an effect of age on levels of gene expression in normal subjects for all genes except HTR2C (Table 2). The same effects of age on histone acetylation and gene expression levels were observed for GAD1, TOMM70A and PPM1E in subjects with bipolar disorder (Table 2); however, in marked contrast, and with the exception of $G A D 1$, there was no effect of age on histone acetylation levels in the prefrontal cortex from subjects with schizophrenia (Table 2).

Histone acetylation differences in young vs old subjects. To further explore the age effect on histone acetylation, we measured ac-H3K9K14 levels at the promoter regions of three of the neuronal genes, GAD1, TOMM70A and HTR2C, plus two oligodendrocyte-expressing genes, myelin basic protein (MBP) and UDP glycosyltransferase 8 (UGT8), and a ubiquitously-expressed gene, $\mathrm{H} 1$ histone family, member $\mathrm{N}$ (H1FNT) in the postmortem prefrontal cortex from a second cohort of subjects (group 2; Supplementary Table 1). This cohort was comprised of young subjects (18-36 years of age) and old subjects (55-92 years of age) with schizophrenia and age-matched controls ( $n=32$ in total). Consistent with the results from subjects in group 1 above, Pearson's correlation analysis of ac-H3K9K14 levels against age revealed strong negative correlation with age in normal subjects (Table 2; Figure 3), but not in subjects with schizophrenia, despite measuring levels in a cohort of subjects with a greater age range (18-91 years). Examining our previous microarray data
HTR2C

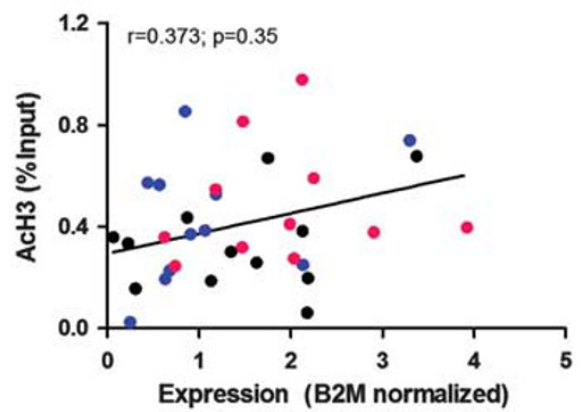

GAD1

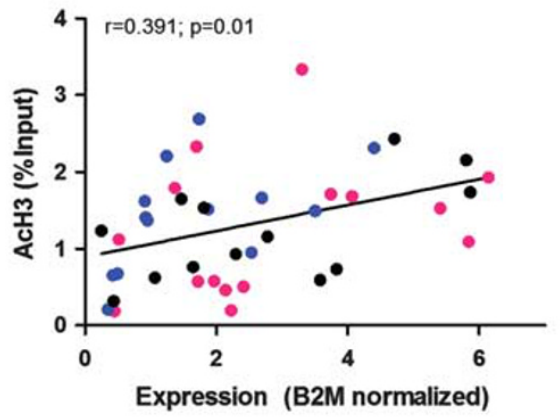

- Normal

- Schizophrenia

- Bipolar disorder
RGS4

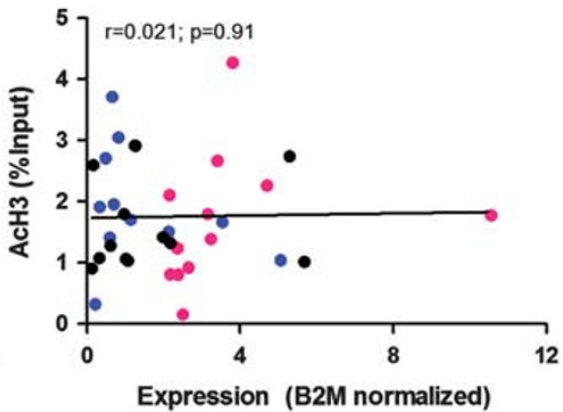

TOMM70A

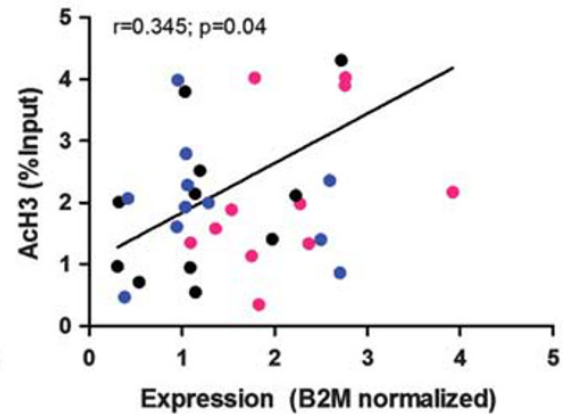

Figure 2 Correlation between gene expression levels and acetylation of histone $\mathrm{H} 3$ at $\mathrm{K} 9$ and $\mathrm{K} 14$. ChIP-qPCR assays were performed on postmortem BA10 from the same subjects as in Figure 1, measuring ac-H3K9K14 levels at the promoter regions of the indicated genes (as designated by UniGene IDs). Rabbit lgG was used as a negative control for the pull-down. Histone acetylation is presented as percentage input DNA. Gene expression levels were determined by real-time qPCR, from Figure 1. Each point represents one subject. Pink, control subjects; blue, subjects with schizophrenia; black, subjects with bipolar disorder. Pearson's correlation ( $r$ ) values are indicated within each graph. 
generated from the prefrontal cortex from case and control subjects ranging in age from 18-81 years (GEO accession \#GSE21138), ${ }^{30,37}$ which consisted of one-half of the same young subjects as used in this study, we similarly find that the expression levels of these genes decreases with age (Supplementary Figure 1).

From the linear plots shown in Figure 3, it is apparent that for several genes, ac-H3K9K14 levels do not decrease with advanced age because levels are low in subjects with schizophrenia at an early age, and remain low throughout aging. Hence, we performed group-wise comparisons of the ChIP-qPCR data according to age. This analysis revealed hypoacetylation of $\mathrm{H} 3 \mathrm{~K} 9 \mathrm{~K} 14$, the promoter regions of $G A D 1$, UGT8, HTR2C and H1FNT in young subjects with schizophrenia compared with matched controls (Figure 4A). In contrast, only HTR2C showed a decrease in ac-H3K9K14 levels in old subjects compared with matched controls, although this did not reach significance $(P=0.071)$ (Figure 4A). Interestingly, ac-H3K9K14 levels at the MBP promoter were significantly increased in old subjects compared with matched controls (Figure 4A).

Again, we examined whether the expression of these genes from our previous microarray studies (GEO accession \#GSE21138), which were performed on the prefrontal cortex from one-half of the same young subjects, ${ }^{30}$ was associated with ac-H3K9K14 levels. We also examined gene expression from subjects at late stage, although they were different than those used for ChIP-qPCR in the current study. Consistent with the observed hypoacetylation of H3K9K14 in young subjects with schizophrenia, we find that the expression of GAD1, TOMM7OA, HTR2C, MBP, UGT8 and H1FNT are decreased in young-aged subjects compared with agematched controls (Figure 4B). Old-aged subjects with schizophrenia compared with their age-matched controls showed no significant changes in expression of these genes, consistent with the lack of difference in ac-H3K9K14 levels in older subjects (Figure 4B).

HDAC inhibitors and schizophrenia candidate genes. The role of histone acetylation on gene regulation is especially pertinent because of the therapeutic potential of HDAC inhibitors, which have gained considerable attention as a relevant therapeutic option for many neurological disorders ${ }^{38,39}$ including psychiatric disorders. ${ }^{5,40}$ Our previous studies have focused on novel, HDAC1/3-selective HDAC inhibitors, including HDACi $\mathbf{4 b} .^{25,41}$ To gain insight into the potential usefulness of novel selective HDAC inhibitors, such as HDACi 4b, we screened our previously published microarray data from HDACi 4b-treated mouse brain $^{25}$ (GEO accession \#GSE26317) for schizophrenia candidate genes as determined from the SZGene database (www.szgene.org). We found that HDACi $\mathbf{4 b}$ treatment altered the expression of several candidate genes for schizophrenia; from the top 45 candidate genes listed on the SZGene database, 17 genes, including RGS4 and MBP, two genes from this study, were found to be altered in the mouse brain by $\mathbf{4 b}$ (Figure 5). This is a significant overrepresentation of $\mathbf{4 b}$-regulated candidate genes than would be expected by chance (Fisher's exact test; $P=0.02$ ). For most of these genes, which have been shown to 

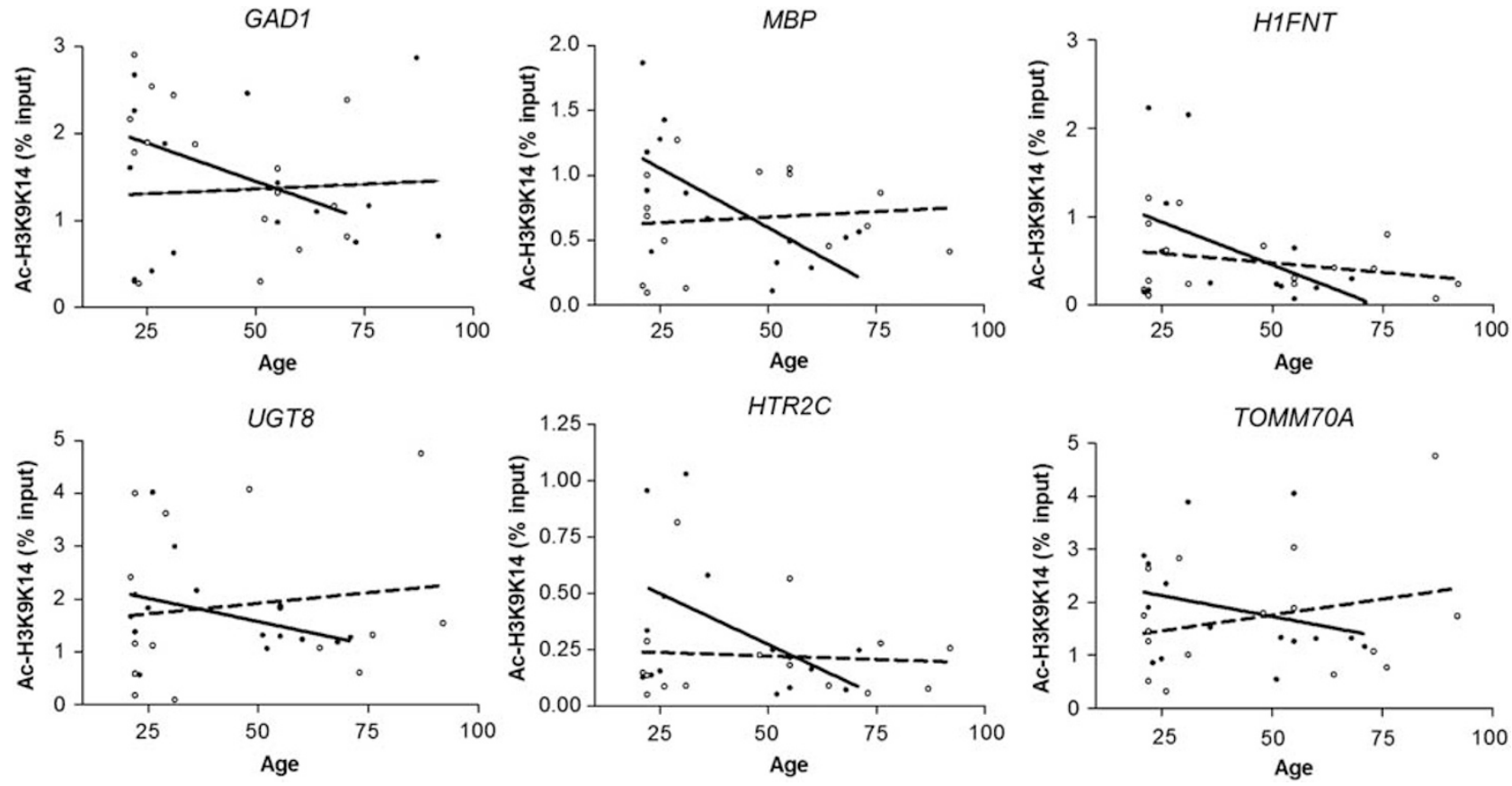

Figure 3 Ac-H3K9K14 levels as a function of age in control subjects and those with schizophrenia. ChIP-qPCR assays were performed on postmortem BA46 from control subjects (closed circles, solid line) and those with schizophrenia (open circles, dashed line) representing a wide age range (group 2; $n=32$ subjects in total). Pearson's ( $r$ ) values are shown in Table 2.

a

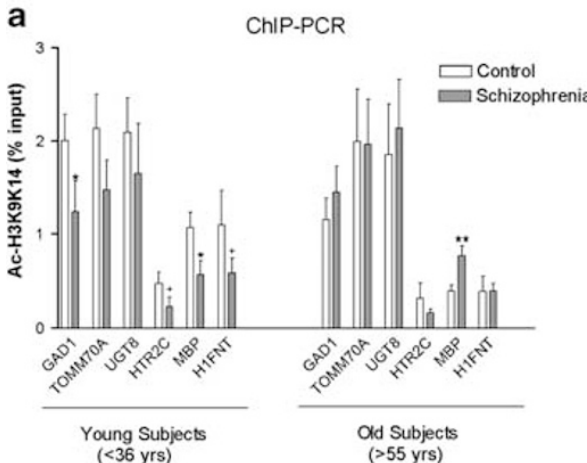

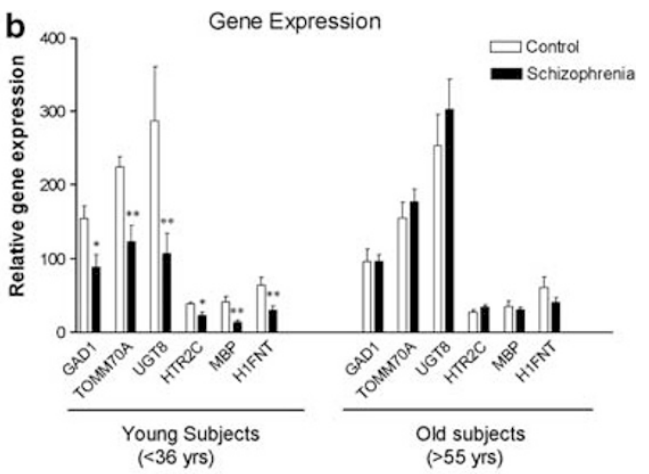

Figure 4 Histone H3K9K14 is hypoacetylated at the promoter regions of genes in young subjects with schizophrenia and associated with decreased gene expression levels. (a) ChIP-qPCR assays were performed on young and old subjects with schizophrenia and age-matched controls (group 2; $n=32$ subjects in total), measuring acH3K9K14 levels at the promoter regions of the indicated genes (UniGene IDs). Asterisks denote significant differences in ac-H3K9K14 levels, as determined by Student's $t$-test: ${ }^{*}, P<0.05,{ }^{+}, P<0.08$. (b) Significant differences in microarray expression values were determined by ANOVA as described in Materials and methods; ${ }^{*}, P<0.05,{ }^{*}$, $P<0.01$.

be decreased in expression in schizophrenia, HDACi $\mathbf{4 b}$ caused an elevation of gene expression (Figure 5).

\section{Discussion}

In this study, we measured gene expression and promoterassociated histone ac-H3K9K14 levels in human postmortem cortex for eight genes representing diverse functions associated with schizophrenia in order to assess the role of epigenetic mechanisms on gene activity. In particular, we included assessment of GAD1, which encodes the 67-kDa glutamate decarboxylase GABA synthesis enzyme. Deficits in the expression of $G A D 1$ are considered to be among the most frequently replicated findings in schizophrenia postmortem brain ${ }^{42,43}$ (reviewed in ref. 44). The major findings from this study are: (1) histone ac-H3K9K14 levels are correlated with gene expression levels for several schizophrenia-related genes, including GAD1; (2) age is strongly negatively associated with promoter-associated histone acetylation levels in normal subjects and those with bipolar disorder, but not schizophrenia and (3) histone H3K9K14 levels are hypoacetylated at the promoter regions of important genes in young subjects with schizophrenia.

Epigenetic mechanisms of gene regulation involve both DNA methylation and an array of posttranslational modifications of histone proteins. ${ }^{1}$ Although DNA methylation has been more 


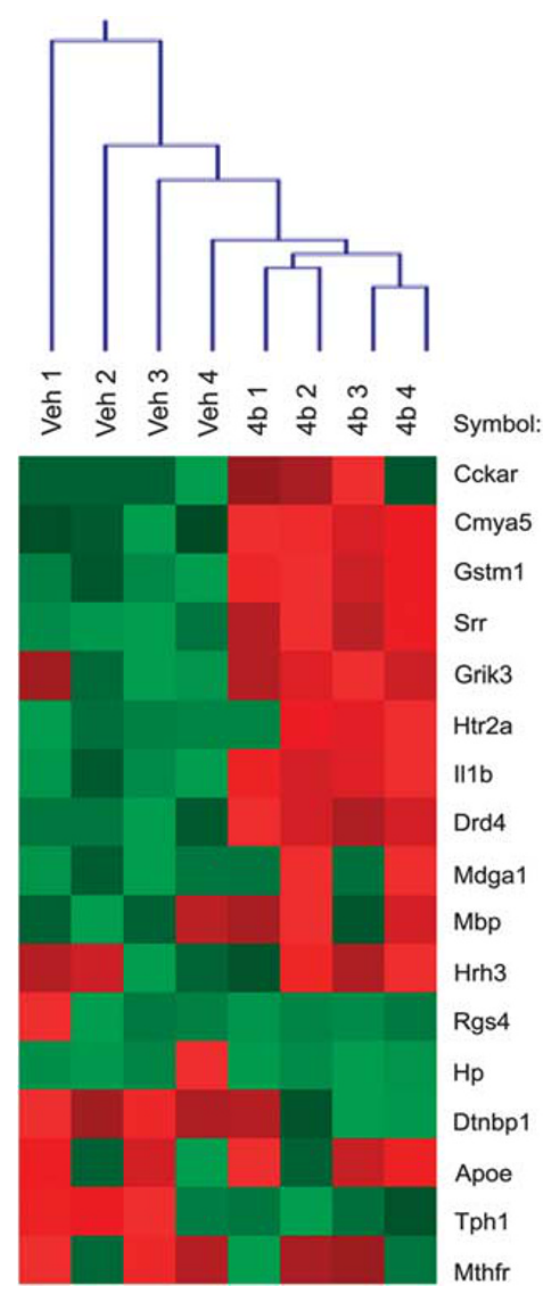

Figure 5 Heatmap depiction of the 17 schizophrenia candidate genes found to be significantly regulated by HDACi $\mathbf{4 b}$ treatment in the mouse brain. Official UniGene symbols are shown for each gene. Each colored pixel represents an individual gene expression value. Relative decreases in gene expression are indicated by green and increases in expression by red. Two-dimensional hierarchical clustering of the samples is shown along the top.

widely studied in the context of psychiatric disorders, in this study we focused on histone acetylation at two specific lysine residues, $\mathrm{K} 9$ and $\mathrm{K} 14$. We demonstrated correlation of acH3K9K14 levels with expression levels of selected genes in the postmortem human prefrontal cortex. These findings are consistent with previous studies showing that acetylation of histone $\mathrm{H} 3$ at $\mathrm{K} 9$ and $\mathrm{K} 14$ are positively correlated with one another and associated with transcriptional activity across a majority of yeast genes. ${ }^{21}$ Epigenetic studies in yeast have also found that ac-H3K9 and ac-H3K14 levels are correlated with levels of trimethylated $\mathrm{H} 3 \mathrm{~K} 4$ (H3K4me3), another epigenetic mark associated with active gene transcription and abundant at the transcription start sites of genes. Genome-wide maps of histone H3K4me3 have been previously identified in the human prefrontal cortex ${ }^{45}$ and these data are freely available on the UCSC web browser (http:// genome.ucsc.edu). Again, consistent with the findings from yeast, we found that the promoter loci bearing ac-H3K9K14 marks for GAD1, RGS4, HTR2C, PPM1E and UGT8 also harbor $\mathrm{H} 3 \mathrm{~K} 4 \mathrm{me} 3$ marks in the human prefrontal cortex. An example of this overlap is shown for UGT8 in Supplementary Figure 2.

The second major finding from this study is that age is strongly negatively correlated with promoter-associated histone acetylation levels in normal subjects. Normal aging is known to be accompanied by genomic instability and changes in gene expression, ${ }^{46}$ and evidence now suggests that epigenetic factors are a major cause of these age-related changes in mice and humans. ${ }^{47,48}$ Most epigenetic studies of the aging brain have focused on DNA methylation where positive correlations between DNA methylation and chronological age have been demonstrated for selected genes, such as GAD1, ${ }^{49}$ as well as genome wide. ${ }^{47-49}$ However, information on how histone modifications change with age is more limited. ${ }^{50}$ Here, we have shown that histone acetylation levels are negatively correlated with age at several gene promoters, including GAD1, RGS4, HTR2C, PPM1E and $M B P$ and that the expression levels of these genes are similarly negatively correlated with age. The gene expression data are consistent with a previous study showing that the expression of several schizophrenia candidate genes, including RGS4 and GAD1, decreases with age in the postmortem prefrontal cortex from normal individuals. ${ }^{51}$ We also found that promoter-associated histone acetylation levels were significantly negatively correlated with age in subjects with bipolar disorder, but not schizophrenia, indicating disease-specific effects of epigenetic gene regulation. We further show that these effects are not unique to cell type-specific gene promoters, as acetylation changes were detected in both neuron- and glia-expressed genes.

The mechanism of the reduced site-specific acetylation with age is unclear; however, a few possibilities could be considered. Altered acetylation levels of histones could occur by changes in the activities of HDAC enzymes. For example, a decrease in HDAC activity has been observed in normal rat liver with increasing age. ${ }^{52}$ Another possibility is that acetylated histones are replaced by newly synthesized unmodified ones. Although it has been shown that histone turnover in the brain is slow, ${ }^{53}$ it could be potentially substantial with aging. It is also possible that some histone modifications decay with time at the promoters of genes that are not active in aged individuals. The lack of an age effect on histone acetylation observed in the brains of subjects with schizophrenia could be due to abnormalities in any of the above-mentioned mechanisms.

Thirdly, we demonstrated that histone H3 is hypoacetylated in young subjects with schizophrenia when compared with age-matched controls. Such hypoacetylation of histone proteins could be reversed by the actions of HDAC inhibitors, thereby improving the associated gene expression deficits. To date, 18 human HDAC subtypes have been identified, which can be divided into four main groups, classes I-IV. ${ }^{38}$ Valproic acid, an inhibitor of class I HDACs, ${ }^{54}$ has a long and established history of efficacy in the treatment of bipolar disorder. Reports have further shown that typical and atypical antipsychotics are more potent, more efficacious and less toxic if they are co-administered with valproic acid, ${ }^{55-57}$ although, some studies did not report such benefit. ${ }^{5-60}$ 
Nonetheless, the beneficial effects of valproic acid that were observed in schizophrenia suggest that more potent and/or more selective HDAC inhibitors may represent a new opportunity for pharmacological interventions for this disorder. Consistent with this view, previous studies have shown that another class I HDAC inhibitor, MS-275, potently activates GAD1 gene expression in NT2 cells accompanied by decreased $G A D 1$ promoter methylation, ${ }^{10}$ and in the current study, we have shown that HDACi $\mathbf{4 b}$ altered the levels of 17 schizophrenia candidate genes in the mouse brain (see Figure 5). Consistent with these findings, previous studies have demonstrated that inhibition of the class I HDACs, HDAC2 and HDAC3, enhances cognition and memory function in rodents. ${ }^{61,62}$

One final note is that the similarity between histone hypoacetylation observed with normal aging and in young subjects with schizophrenia is consistent with emerging data showing phenotypic overlap between normal aging and earlystage schizophrenia. Normal aging has been linked to alterations in white matter density and volume, gray matter volume decline, cognitive dysfunction, shortened telomeres, microglia activation and psychotic symptoms, ${ }^{63-66}$ which also characterize schizophrenia at first episode or recent onset. ${ }^{67-71}$ Furthermore, our own previous studies have demonstrated that normal human aging and early-stage schizophrenia share common molecular phenotypes. ${ }^{37}$

In summary, our data demonstrate that gene expression changes associated with psychiatric disease and aging result from epigenetic mechanisms of gene regulation involving histone acetylation. These findings provide a relevant basis for the use of HDAC inhibitors as therapeutic treatment for schizophrenia, particularly in young subjects (that is, $<36$ years of age), whereby the use of HDAC inhibitors may be therapeutically beneficial by means of restoring abnormal histone acetylation patterns and accompanying gene expression deficits in schizophrenia leading to improved clinical symptoms. Similarly, HDAC inhibitors may also be useful for treatment of age-related phenotypes, such as psychosis and cognitive decline, which are similar to those typically observed in subjects with schizophrenia.

\section{Conflict of interest}

The authors declare no conflict of interest.

Acknowledgements. This study was funded by Grants from the National Institutes of Health (NS44169 and MH069696 to E.A.T.). Tissues used in this study were provided in part by the Harvard Brain Tissue Resource Center, which is supported in part by PHS Grant \#R24 MH068855. Other tissues were received from the Victorian Brain Bank Network, supported by the Mental Health Research Institute, Alfred Hospital, Victorian Forensic Institute of Medicine and The University of Melbourne. B.D. is a NHMRC Senior Research Follow (APP1002240).

1. Quina AS, Buschbeck M, Di Croce L. Chromatin structure and epigenetics. Biochem Pharmacol 2006; 72: 1563-1569.

2. Attwood JT, Yung RL, Richardson BC. DNA methylation and the regulation of gene transcription. Cell Mol Life Sci 2002; 59: 241-257.

3. Kouzarides T. Chromatin modifications and their function. Cell 2007; 128: 693-705.

4. Akbarian S. The molecular pathology of schizophrenia-focus on histone and DNA modifications. Brain Res Bull 2010; 83: 103-107.
5. Deutsch SI, Rosse RB, Mastropaolo J, Long KD, Gaskins BL. Epigenetic therapeutic strategies for the treatment of neuropsychiatric disorders: ready for prime time? Clin Neuropharmacol 2008; 31: 104-119.

6. Roth TL, Lubin FD, Sodhi M, Kleinman JE. Epigenetic mechanisms in schizophrenia. Biochim Biophys Acta 2009; 1790: 869-877.

7. Tsankova N, Renthal W, Kumar A, Nestler EJ. Epigenetic regulation in psychiatric disorders. Nat Rev Neurosci 2007; 8: 355-367.

8. Carrard A, Salzmann A, Malafosse A, Karege F. Increased DNA methylation status of the serotonin receptor $5 \mathrm{HTR} 1 \mathrm{~A}$ gene promoter in schizophrenia and bipolar disorder. J Affect Disord 2011; 132: 450-453

9. Abdolmaleky HM, Yaqubi S, Papageorgis P, Lambert AW, Ozturk S, Sivaraman V et al. Epigenetic dysregulation of HTR2A in the brain of patients with schizophrenia and bipolar disorder. Schizophr Res 2011; 129: 183-190.

10. Chen $\mathrm{Y}$, Dong $\mathrm{E}$, Grayson DR. Analysis of the GAD1 promoter: trans-acting factors and DNA methylation converge on the $5^{\prime}$ untranslated region. Neuropharmacology 2011; 60: 1075-1087.

11. Huang HS, Akbarian S. GAD1 mRNA expression and DNA methylation in prefrontal cortex of subjects with schizophrenia. PLOS One 2007; 2: e809.

12. Abdolmaleky HM, Cheng KH, Russo A, Smith $\mathrm{CL}$, Faraone SV, Wilcox $\mathrm{M}$ et al. Hypermethylation of the reelin (RELN) promoter in the brain of schizophrenic patients: a preliminary report. Am J Med Genet B Neuropsychiatr Genet 2005; 134B: 60-66.

13. Grayson DR, Jia X, Chen $Y$, Sharma RP, Mitchell $C P$, Guidotti $A$ et al. Reelin promoter hypermethylation in schizophrenia. Proc Natl Acad Sci U S A 2005; 102 . 9341-9346.

14. Abdolmaleky HM, Cheng KH, Faraone SV, Wilcox M, Glatt SJ, Gao F et al. Hypomethylation of MB-COMT promoter is a major risk factor for schizophrenia and bipolar disorder. Hum Mol Genet 2006; 15: 3132-3145.

15. Petronis A, Gottesman II, Kan P, Kennedy JL, Basile VS, Paterson AD et al. Monozygotic twins exhibit numerous epigenetic differences: clues to twin discordance? Schizophr Bull 2003; 29: 169-178.

16. Iwamoto K, Bundo M, Yamada K, Takao H, Iwayama-Shigeno Y, Yoshikawa T et al. DNA methylation status of SOX10 correlates with its downregulation and oligodendrocyte dysfunction in schizophrenia. J Neurosci 2005; 25: 5376-5381.

17. Mill J, Tang T, Kaminsky Z, Khare T, Yazdanpanah S, Bouchard L et al. Epigenomic profiling reveals DNA-methylation changes associated with major psychosis. Am J Hum Genet 2008; 82: 696-711.

18. Akbarian S, Ruehl MG, Bliven E, Luiz LA, Peranelli AC, Baker SP et al. Chromatin alterations associated with down-regulated metabolic gene expression in the prefrontal cortex of subjects with schizophrenia. Arch Gen Psychiatry 2005; 62: 829-840.

19. Mirnics K, Levitt $P$, Lewis DA. Critical appraisal of DNA microarrays in psychiatric genomics. Biol Psychiatry 2006; 60: 163-176.

20. Thomas EA. Molecular profiling of antipsychotic drug function: convergent mechanisms in the pathology and treatment of psychiatric disorders. Mol Neurobiol 2006; 34: 109-128.

21. Pokholok DK, Harbison CT, Levine S, Cole M, Hannett NM, Lee TI et al. Genome-wide map of nucleosome acetylation and methylation in yeast. Cell 2005; 122: 517-527.

22. Hill C, Keks NA, Roberts S, Opeskin K, Dean B, Copolov DL. Postmortem brain studies in schizophrenia: the problems of diagnosis. Am J Psychiatry 1996; 153: 533-537.

23. Stan AD, Ghose S, Gao XM, Roberts RC, Lewis-Amezcua K, Hatanpaa KJ et al. Human postmortem tissue: what quality markers matter? Brain Res 2006; 1123: 1-11.

24. Luo RX, Postigo AA, Dean DC. Rb interacts with histone deacetylase to repress transcription. Cell 1998; 92: 463-473.

25. Thomas EA, Coppola G, Desplats PA, Tang B, Soragni E, Burnett R et al. The HDAC inhibitor, $4 b$, ameliorates the disease phenotype and transcriptional abnormalities in Huntington's disease transgenic mice. Proc Natl Acad Sci U S A 2008; 105: 15564-15569.

26. Desplats PA, Kass KE, Gilmartin T, Stanwood GD, Woodward EL, Head SR et al. Selective deficits in the expression of striatal-enriched mRNAs in Huntington's disease. J Neurochem 2006; 96: 743-757.

27. Narayan S, Kass K, Thomas EA. Chronic haloperidol treatment results in a decrease in the expression of myelin/oligodendrocyte-related genes in the mouse brain. J Neurosci Res 2006; 85: 757-765.

28. Sharov AA, Dudekula DB, Ko MS. A web-based tool for principal component and significance analysis of microarray data. Bioinformatics 2005; 21: 2548-2549.

29. Benjamini $Y$, Hochberg $Y$. Controlling the false discovery rate-a practical and powerful approach to multiple testing. J $R$ Stat Soc B 1995; 57: 289-300.

30. Narayan S, Tang B, Head SR, Gilmartin TJ, Sutcliffe JG, Dean B et al. Molecular profiles of schizophrenia in the CNS at different stages of illness. Brain Res 2008; 1239: 235-248.

31. Kim S, Webster MJ. The stanley neuropathology consortium integrative database: a novel, web-based tool for exploring neuropathological markers in psychiatric disorders and the biological processes associated with abnormalities of those markers. Neuropsychopharmacology 2010; 35: 473-482.

32. Cahoy JD, Emery B, Kaushal A, Foo LC, Zamanian JL, Christopherson KS et al. A transcriptome database for astrocytes, neurons, and oligodendrocytes: a new resource for understanding brain development and function. J Neurosci 2008; 28: 264-278.

33. Mirnics K, Middleton FA, Marquez A, Lewis DA, Levitt P. Molecular characterization of schizophrenia viewed by microarray analysis of gene expression in prefrontal cortex. Neuron 2000; 28: 53-67. 
34. Akbarian S, Huang HS. Molecular and cellular mechanisms of altered GAD1/GAD67 expression in schizophrenia and related disorders. Brain Res Rev 2006; 52: 293-304.

35. Marazziti D, Baroni S, Picchetti M, Landi P, Silvestri S, Vatteroni E et al. Mitochondria alterations and neuropsychiatric disorder. Curr Med Chem 2011; 18: 4715-4721.

36. Torkamani A, Dean B, Schork NJ, Thomas EA. Coexpression network analysis of neural tissue reveals perturbations in developmental processes in schizophrenia. Genome Res 2010; 20: 403-412.

37. Tang B, Chang WL, Lanigan CM, Dean B, Sutcliffe JG, Thomas EA. Normal human aging and early-stage schizophrenia share common molecular profiles. Aging Cell 2009; 8: 339-342.

38. Thomas EA. Focal nature of neurological disorders necessitates isotype-selective histone deacetylase (HDAC) inhibitors. Mol Neurobiol 2009; 40: 33-45.

39. Kazantsev AG, Thompson LM. Therapeutic application of histone deacetylase inhibitors for central nervous system disorders. Nat Rev Drug Discov 2008; 7: 854-868.

40. Grayson DR, Kundakovic M, Sharma RP. Is there a future for histone deacetylase inhibitors in the pharmacotherapy of psychiatric disorders? Mol Pharmacol 2010; 77: 126-135.

41. Chou CJ, Herman D, Gottesfeld JM. Pimelic diphenylamide 106 is a slow, tight-binding inhibitor of class I histone deacetylases. J Biol Chem 2008; 283: 35402-35409.

42. Akbarian S, Huntsman MM, Kim JJ, Tafazzoli A, Potkin SG, Bunney Jr WE et al. GABAA receptor subunit gene expression in human prefrontal cortex: comparison of schizophrenics and controls. Cereb Cortex 1995; 5: 550-560.

43. Akbarian S, Kim JJ, Potkin SG, Hagman JO, Tafazzoli A, Bunney Jr WE et al. Gene expression for glutamic acid decarboxylase is reduced without loss of neurons in prefrontal cortex of schizophrenics [see comments]. Arch Gen Psychiatry 1995; 52: 258-266.

44. Guidotti A, Auta J, Davis JM, Dong E, Grayson DR, Veldic M et al. GABAergic dysfunction in schizophrenia: new treatment strategies on the horizon. Psychopharmacology (Berl) 2005; 180: 191-205.

45. Cheung I, Shulha HP, Jiang Y, Matevossian A, Wang J, Weng Z et al. Developmental regulation and individual differences of neuronal $\mathrm{H} 3 \mathrm{~K} 4 \mathrm{me} 3$ epigenomes in the prefrontal cortex. Proc Natl Acad Sci U S A 2010; 107: 8824-8829.

46. Oberdoerffer $P$, Sinclair DA. The role of nuclear architecture in genomic instability and ageing. Nat Rev Mol Cell Biol 2007; 8: 692-702.

47. Hernandez DG, Nalls MA, Gibbs JR, Arepalli S, van der Brug M, Chong S et al. Distinct DNA methylation changes highly correlated with chronological age in the human brain. Hum Mol Genet 2011; 20: 1164-1172.

48. Takasugi M. Progressive age-dependent DNA methylation changes start before adulthood in mouse tissues. Mech Ageing Dev 2011; 132: 65-71.

49. Siegmund KD, Connor CM, Campan M, Long TI, Weisenberger DJ, Biniszkiewicz D et al. DNA methylation in the human cerebral cortex is dynamically regulated throughout the life span and involves differentiated neurons. PLoS One 2007; 2: e895.

50. Sedivy JM, Banumathy G, Adams PD. Aging by epigenetics-a consequence of chromatin damage? Exp Cell Res 2008; 314: 1909-1917.

51. Colantuoni C, Hyde TM, Mitkus S, Joseph A, Sartorius L, Aguirre C et al. Age-related changes in the expression of schizophrenia susceptibility genes in the human prefrontal cortex. Brain Struct Funct 2008; 213: 255-271.

52. Kozurkova M, Misurova $\mathrm{E}$, Kropacova $\mathrm{K}$. Effect of aging and gamma radiation on acetylation of rat liver histones. Mech Ageing Dev 1995; 78: 1-14.

53. Commerford SL, Carsten AL, Cronkite EP. Histone turnover within nonproliferating cells. Proc Natl Acad Sci U S A 1982; 79: 1163-1165.

54. Gurvich N, Tsygankova OM, Meinkoth JL, Klein PS. Histone deacetylase is a target of valproic acid-mediated cellular differentiation. Cancer Res 2004; 64: 1079-1086.

55. Wassef AA, Dott SG, Harris A, Brown A, O'Boyle M, Meyer 3rd WJ et al. Randomized, placebo-controlled pilot study of divalproex sodium in the treatment of acute exacerbations of chronic schizophrenia. J Clin Psychopharmacol 2000; 20: 357-361.

56. Wassef AA, Hafiz NG, Hampton D, Molloy M. Divalproex sodium augmentation of haloperidol in hospitalized patients with schizophrenia: clinical and economic implications. J Clin Psychopharmacol 2001; 21: 21-26.

57. Citrome L. Schizophrenia and valproate. Psychopharmacol Bull 2003; 37(Suppl 2): 74-88.

58. Casey DE, Daniel DG, Wassef AA, Tracy KA, Wozniak P, Sommerville KW. Effect of divalproex combined with olanzapine or risperidone in patients with an acute exacerbation of schizophrenia. Neuropsychopharmacology 2003; 28: 182-192.

59. Citrome L. Adjunctive lithium and anticonvulsants for the treatment of schizophrenia: what is the evidence? Expert Rev Neurother 2009; 9: 55-71.

60. Casey DE, Daniel DG, Tamminga C, Kane JM, Tran-Johnson T, Wozniak P et al. Divalproex ER combined with olanzapine or risperidone for treatment of acute exacerbations of schizophrenia. Neuropsychopharmacology 2009; 34: 1330-1338.

61. Guan JS, Haggarty SJ, Giacometti E, Dannenberg JH, Joseph N, Gao J et al. HDAC2 negatively regulates memory formation and synaptic plasticity. Nature 2009; 459: 55-60.

62. McQuown SC, Barrett RM, Matheos DP, Post RJ, Rogge GA, Alenghat T et al. HDAC3 is a critical negative regulator of long-term memory formation. $J$ Neurosci $2011 ; 31$ : 764-774

63. Yankner BA, Lu T, Loerch P. The aging brain. Annu Rev Pathol 2008; 3: 41-66

64. van der Werf M, van Boxtel M, Verhey F, Jolles J, Thewissen V, van Os J. Mild hearing impairment and psychotic experiences in a normal aging population. Schizophr Res 2007; 94: $180-186$

65. von Zglinicki T, Martin-Ruiz CM. Telomeres as biomarkers for ageing and age-related diseases. Curr Mol Med 2005: 5: 197-203.

66. Miller KR, Streit WJ. The effects of aging, injury and disease on microglial function: a case for cellular senescence. Neuron Glia Biol 2007; 3: 245-253.

67. Lieberman JA. Is schizophrenia a neurodegenerative disorder? A clinical and neurobiological perspective. Biol Psychiatry 1999; 46: 729-739.

68. Witthaus H, Brune M, Kaufmann C, Bohner G, Ozgurdal S, Gudlowski Y et al. White matter abnormalities in subjects at ultra high-risk for schizophrenia and first-episode schizophrenic patients. Schizophr Res 2008; 102: 141-149.

69. Steen RG, Mull C, McClure R, Hamer RM, Lieberman JA. Brain volume in first-episode schizophrenia: systematic review and meta-analysis of magnetic resonance imaging studies. Br J Psychiatry 2006; 188: 510-518.

70. Fernandez-Egea E, Bernardo M, Heaphy CM, Griffith JK, Parellada E, Esmatjes E et al. Telomere length and pulse pressure in newly diagnosed, antipsychotic-naive patients with nonaffective psychosis. Schizophr Bull 2009; 35: 437-442.

71. van Berckel BN, Bossong MG, Boellaard R, Kloet R, Schuitemaker A, Caspers E et al. Microglia activation in recent-onset schizophrenia: a quantitative (R)-[11C]PK11195 positron emission tomography study. Biol Psychiatry 2008; 64: 820-822.

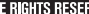

Translational Psychiatry is an open-access journal published by Nature Publishing Group. This work is licensed under the Creative Commons Attribution-Noncommercial-No Derivative Works 3.0 Unported License. To view a copy of this license, visit http://creativecommons.org/licenses/by-nc-nd/3.0/

Supplementary Information accompanies the paper on the Translational Psychiatry website (http://www.nature.com/tp) 